\title{
Enhancing VoIP service for ubiquitous communication in a campus WLAN with partial coverage
}

\author{
Hung-Yun Hsieh ${ }^{\mathrm{a}, \mathrm{b}, *}$, You-En Lin ${ }^{\mathrm{b}}$, Hsiao-Pu Lin ${ }^{\mathrm{b}}$ \\ ${ }^{a}$ Department of Electrical Engineering, National Taiwan University, Taipei 106, Taiwan \\ ${ }^{\mathrm{b}}$ Graduate Institute of Communication Engineering, National Taiwan University, Taipei 106, Taiwan
}

\section{A R T I C L E I N F O}

Available online 24 April 2008

\section{Keywords:}

SIP

PESQ

WSOLA

Vertical handover

Multi-hop relay

Multi-mode handset

\begin{abstract}
A B S T R A C T
The IEEE 802.11 WLAN technology has become the de facto standard for wireless Internet access. The spotty coverage of WLAN access points, however, confines the applicability of many real-time services such as VoIP within the boundary of the WLAN service area. In this paper, we investigate the problem of enhancing VoIP service for ubiquitous communication in a WLAN with spotty service area. We consider a university campus that has an established infrastructure for supporting SIP-based VoIP service through either wired or wireless data networks. The campus WLAN service does not have $100 \%$ full coverage, and hence users cannot make untethered VoIP calls anywhere on campus. The goal of this paper is to overcome the limitations of such "dead spots" for motivating the use of campus IP telephony service. To proceed, we start with two approaches called one-hop extension and dualmode communication. The first approach uses multi-hop relay to extend the WLAN coverage, while the second approach leverages the availability of dual-mode handsets for ubiquitous voice communication. We implement the two approaches, and evaluate their performance in the campus testbed environment. We find that while the two approaches can effectively allow voice communication in WLAN dead spots, they have one common problem as the potential lack of support for voice call continuity that can cause degradation of the speech quality to an active call. We adopt a cross-layer solution based on signal processing algorithms to address the problem, thus achieving seamless voice call continuity while enabling ubiquitous voice communication on campus. Testbed evaluation shows promising results for future research along the proposed direction.
\end{abstract}

(c) 2008 Elsevier B.V. All rights reserved.

\section{Introduction}

VoIP (Voice over IP) over the past few years has successfully ridden on the pervasiveness of Internet infrastructure to extend the reach of telephony service wherever Internet is accessible. Voice over WLAN (VoWLAN) in particular has attracted a lot of attention due to its ability to provide untethered communication while incurring significantly lower cost. Several challenges remain to be addressed,

\footnotetext{
* Corresponding author. Address: Department of Electrical Engineering, National Taiwan University, Room 546, EE-II Building, No. 1, Section 4, Roosevelt Road, Taipei 106, Taiwan. Tel.: +886 233663666.

E-mail address: hyhsieh@cc.ee.ntu.edu.tw (H.-Y. Hsieh).
}

however, in order for VoWLAN to provide service with quality parallel to that provided by mobile cellular telephony. In particular, IEEE 802.11, the de facto standard for WLAN technology, was originally designed without inherent support for QoS required by multimedia services such as VoIP. Problems such as unpredictable delay jitters due to random contention, bursty packet losses due to wireless interference and user mobility, poor scalability, and lack of security have been identified and under investigation by many research endeavors [1-4].

In addition to problems related to the quality, scalability, and security of VoIP calls, another important problem inherent in VoWLAN is the limitation of coverage area. Due to the regulation of transmission power in the unlicensed bands, 
WLAN access points typically have limited transmission range, and hence the WLAN service area is typically spotty. While more and more cities worldwide have undertaken plans to build large-scale wireless mesh networks with the goal of enabling city-wide wireless Internet access $[5,6]$, the coverage still cannot parallel that provided by existing mobile cellular telephony systems. The impact of such spotty coverage is that mobile users are essentially confined to the WLAN service area for making or receiving VoIP calls. Moreover, VoIP calls placed within a WLAN hot spot cannot be carried over to neighboring hot spots without causing call interruptions or even call drops. VoIP users thus need to be constantly aware of the WLAN coverage in order for them to enjoy untethered telephony service. Such restrictions significantly limit the popularity of VoIP service.

In this paper, we investigate the problem of enhancing VoIP service for ubiquitous communication in a WLAN environment with spotty coverage. We start with a case study on a university campus that has an established SIP (Session Initiation Protocol) infrastructure for VoIP service. The campus has provisioned SIP servers including SIP proxies, SIP registrars, and SIP-PSTN gateways that allow users to place VoIP calls with any other phones through campus wired or wireless networks. A wireless user, for example, can place VoIP calls when it is within the coverage of WLAN access points. The campus wireless network we consider, however, does not have $100 \%$ full coverage, and hence VoIP service cannot yet be conveniently used anywhere, anytime on campus.

To motivate the use of VoIP service in such a campus WLAN without letting users worry about unreachable or disrupted calls, we propose the following two approaches that can be used in tandem or alone to address the problem of partial WLAN coverage:

1. The first approach, referred to as one-hop extension, is to extend WLAN coverage through multi-hop communication. When a mobile handset is outside the WLAN coverage, it does not have direct communication with any WLAN access point, but it may be in the neighborhood of nodes with direct WLAN access. Therefore, a conceivable scenario is for nodes within the WLAN service area to relay traffic for those outside. If mobile handsets through multi-hop relay can attain the desired call quality for voice communication without sacrificing the performance of the relaying node, such an approach can effectively extend the VoIP service to areas outside the campus WLAN coverage. While related work has used multi-hop routing protocols for achieving this goal [7-9], in this paper we consider a simple approach for one-hop extension of WLAN access points that does not incur the overheads of conventional multi-hop routing protocols.

2. The second approach, referred to as dual-mode communication, is to leverage the capability of mobile handsets with dual modes of communication including GSM${ }^{1}$ and WLAN modes. With the availability of dual communica-

\footnotetext{
1 We use GSM as a general term to refer to mobile cellular telephony systems, including PCS, IS-95, UMTS, and CDMA2000.
}

tion modes, when a mobile user in a VoWLAN call moves beyond the coverage of WLAN service, the call is transferred automatically to the GSM mode for uninterrupted communication with the remote peer. When the mobile user moves back to the WLAN coverage, on the other hand, the GSM call is transferred back to the WLAN mode. If such call transfer can be performed seamlessly without causing undesirable call disruptions, users are motivated to use VoIP service whenever possible since the cost of the call can be significantly reduced without sacrificing reliability. While related work has investigated infrastructure-based approaches for achieving this goal [10-13], in this paper we consider an end-to-end approach that does not require the infrastructure support from the telecom service provider.

We evaluate the proposed approaches through testbed implementation and measurement. We show for the first approach that one-hop extension can effectively provide VoIP service to users outside the campus WLAN coverage without significantly degrading the call quality of the relaying node. However, there might be a transient increase in delay jitter on the relaying node at the onset of relay that can potentially introduce packet losses. For the second approach, we show that it is possible to transfer an active call between GSM and WLAN modes on the dual-mode handset as needed. Due to the delay mismatch between GSM and VoWLAN calls, however, there might be an audio gap during the handoff process.

Our evaluation results show that it is possible to provide ubiquitous voice communication for VoIP users in a campus WLAN with partial coverage through one-hop extension, dual-mode communication, or combination thereof. To provide ubiquitous yet still seamless voice communication, however, the problems of delay surge in one-hop extension and delay mismatch in dual-mode communication need to be addressed. To proceed, we propose a cross-layer solution based on signal processing algorithms to ensure seamless voice call continuity when the proposed approaches are used. Essentially, the delay surge of VoWLAN packets and the delay mismatch of VoWLAN and GSM calls introduce temporal discontinuity (audio gap) to the received voice stream on the relaying node and the dual-mode handset, respectively. We propose the use of time-scale modification of speech to mitigate such temporal discontinuity and remove the unpleasant call disruption to users. Evaluation results show that time-scale modification of speech can address the problems in one-hop extension and dual-mode communication, and significantly improves user experience in this context.

It has to be noted that while we focus on a campus WLAN for a case study of the target problem, the proposed solutions can also be applied in other environments such as enterprise and community networks with similar settings. The contribution of this paper therefore is twofold:

1. We propose and evaluate through testbed experiments two approaches, one-hop extension and dual-mode communication, that can be used in tandem or alone to enhance VoIP service for ubiquitous voice communication in a WLAN with partial coverage. 
2. We propose a cross-layer solution based on time-scale modification of speech that can be used in the proposed two approaches to address the problem of temporal discontinuity for achieving seamless voice continuity while enabling ubiquitous voice communication.

The rest of the paper is organized as follows. Section 2 introduces the campus WLAN that we consider in this paper, and presents some baseline performance for VoIP service in the target environment. Section 3 presents the first approach based on one-hop extension, and Section 4 presents the second approach based on dual-mode communication. Section 5 proposes and evaluates the signal processing algorithm to be used in tandem with the two approaches for seamless voice call continuity. Finally, Section 6 concludes the paper.

\section{NTU campus wireless LAN}

In this section, we first describe the network environment on National Taiwan University (NTU) campus, including SIP infrastructure and the WLAN coverage. We then present measurement results obtained at different locations of the campus that we use later in this paper as the baseline VoIP performance.

\subsection{Network infrastructure}

The National Taiwan University main campus is located at downtown Taipei city, covering an area of 1.129 square kilometers while hosting a population of about 32,000 students. The campus network is based on
$100 \mathrm{Mbps}$ Fast Ethernet, with the core backbone running at the speed of $10 \mathrm{Gbps}$ Gigabit Ethernet. Fig. 1 shows the network architecture at National Taiwan University, with focus on related SIP infrastructure including the call server and media gateway. The call server includes the proxy server, registration server, and location server for performing various SIP related functionalities for SIP based voice calls. The media gateway system supports SIP-to-PBX and SIP-to-PSTN calls between SIP phones (or softphones) and campus PBX or city PSTN phones. SIP phones can connect to the NTU SIP network through either campus WLAN or individual LANs installed at each building. Currently, several campus residence halls have been upgraded by replacing PBX phones with SIP phones, and SIP based phone systems have been under operation in several administrative buildings. Several projects and trials have also been launched for promoting campuswide IP telephony among staff, faculty, and students, including the distribution and application of wireless SIP phones.

For a user without wired connection, the campus WLAN can be used for connecting to the SIP infrastructure. NTU has a rather mature campus WLAN deployment, with an estimated coverage of over $80 \%$ campus area as shown in Fig. 2. As mature as the NTU campus WLAN is, however, it can be observed from the figure that there are locations where the WLAN signal is either low and unstable or totally not present due to obstruction of buildings/trees and insufficient deployment density. While the WLAN coverage can indeed be increased by deploying more access points at more campus sites, over time the coverage of the NTU campus WLAN has not changed significantly since

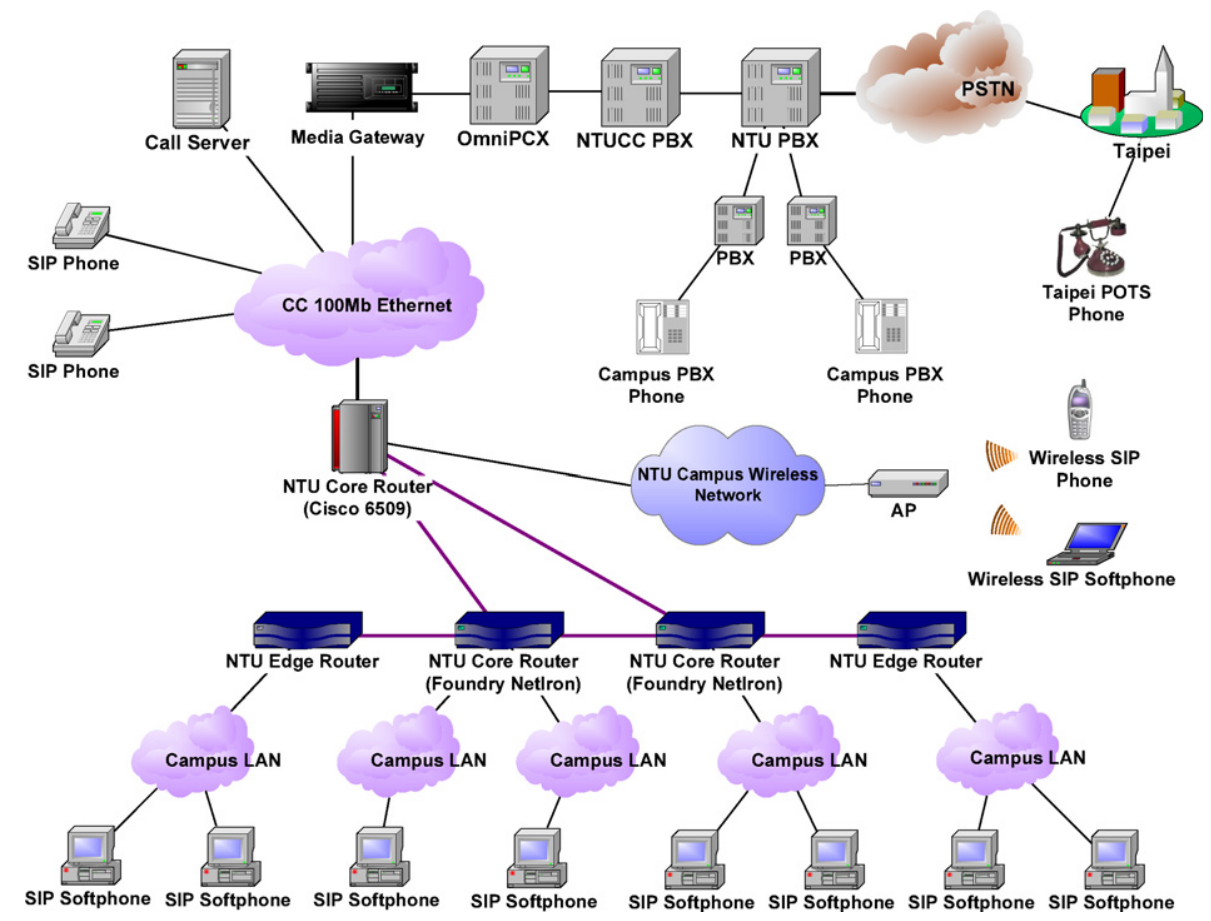

Fig. 1. NTU SIP infrastructure. 


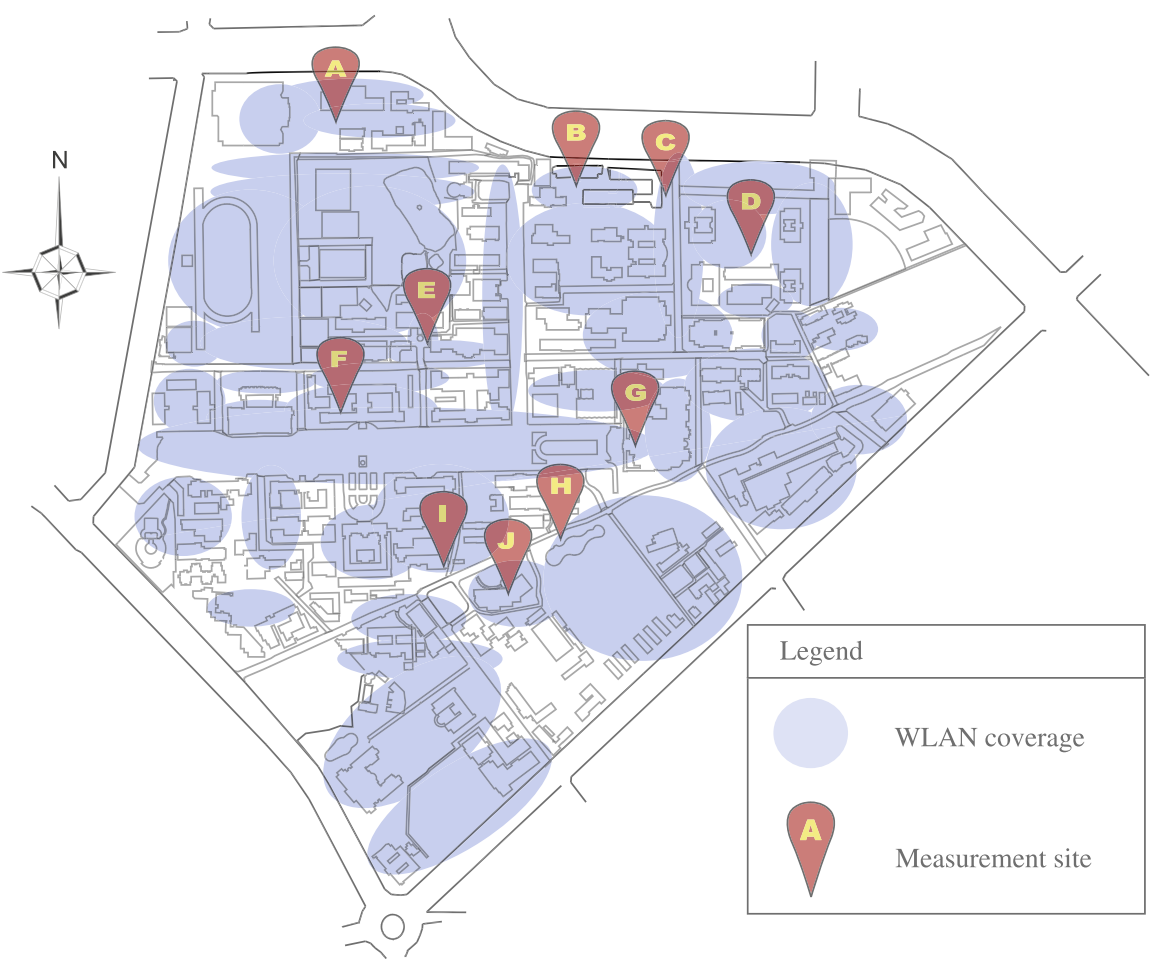

Fig. 2. NTU campus WLAN coverage.

it has reached its maturity. The reason is that the deployment strategy of WLAN access points on campus typically depends on many considerations such as site popularity, usage pattern, and maintenance efforts, rather than on the sole purpose of $100 \%$ full coverage. That being the case, users of portable laptops may still enjoy being able to access Internet at selected outdoor locations on campus. For users of mobile phones who may make calls while on the go, however, it may not be motivating to use a telephony system that is not available when needed - or even worse, that may potentially disconnect calls during the course of mobility. It is therefore the goal of this paper to propose solutions that can overcome the limitations of insufficient WLAN coverage for enabling ubiquitous voice communication on campus and motivating the use of campus VoIP service. The proposed solutions leverage the large student base and the capability of mobile handsets instead of asking for more infrastructure support as we show later in this paper.

\subsection{Baseline performance}

We select 10 different locations on NTU campus to measure the performance of the campus WLAN in supporting VoIP traffic. As shown in Fig. 2, we measure at indoor and outdoor locations that are likely to have heavy traffic load as well as locations that are not. To understand the baseline performance of the campus WLAN for VoIP service, we conduct experiments to measure the network characteristics and VoIP service quality:
1. To proceed with the experiment on network characteristics, we first capture the traffic sent out by a SIP softphone and analyze the average packet size as well as the packet interval [14]. A traffic generator is then used to generate traffic that is an emulation of the measured VoIP traffic. For each packet sent out by the traffic generator, the sending time is stamped in the packet header to be echoed back by the receiver for round-trip time measurement. Multiple runs are conducted at each location and the round-trip time (maximum, minimum, and average) and delay jitter as well as loss rate are obtained for further analysis.

2. To proceed with the experiment on VoIP service quality, we first record a spontaneous speech of about 80s uttered by a female speaker. At one SIP softphone, the recorded speech is played back automatically when a call is established. Another SIP softphone is used to make the voice call and record the voice stream that it receives from the peer. The received speech is compared against the original speech for evaluation of the speech quality. We use PESQ (Perceptual Evaluation of Speech Quality) [15] as the metric for measuring the speech quality, and hence VoIP service quality, at the campus site. PESQ is an objective method for end-toend speech quality assessment as recommended by ITU. Unlike the $R$ Factor (Transmission Rating Factor) from the E-Model [16], the calculation of the PESQ score does not rely on the transformations of various transmission parameters to speech impairment factors for predicting the quality of the call. Instead, the PESQ 
model compares an original signal $x(n)$ with a degraded signal $y(n)$ using human perceptual and cognitive models [15]. The PESQ score ranges from -0.5 (worst) to 4.5 (best), which has been mapped through regression to the MOS (Mean Opinion Score) subjective score using a large set of subjective experiments.

Since the goal of this paper is not to conduct a comprehensive performance measurement and analysis of the campus WLAN, we show in Fig. 3 a subset of measurement data obtained at different campus sites. It can be observed from the figure that at all locations selected the average round-trip time is less than $210 \mathrm{~ms}$, and the delay jitter is less than $70 \mathrm{~ms}$. Locations $\mathrm{A}, \mathrm{F}$, and I have measured loss rates of more than $2 \%$ due to poor signal quality and/or heavy traffic load. Note that latency, jitter, and loss rate all play important roles in determining the quality of the call. However, as Fig. 3d shows, overall VoIP service quality at all locations is rather acceptable. Therefore, if the problem of partial WLAN coverage can be addressed, the campus WLAN can potentially be a good carrier for supporting untethered IP telephony.

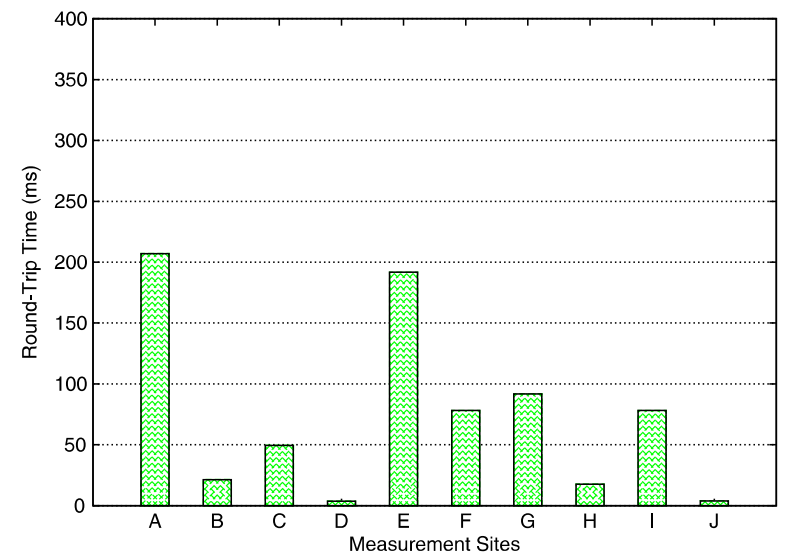

(a) Round-TripTime

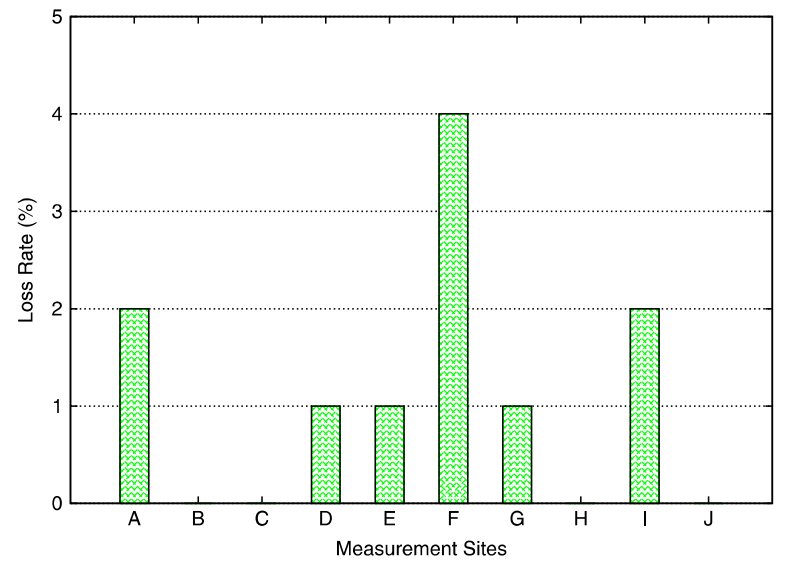

(c) Loss Rate

\section{One-hop extension}

In this section we present the first approach to extend VoIP service to the dead spots of the campus WLAN. We first motivate the solution based on onehop extension, and then present the proposed approach. Finally, we present evaluation results and some discussions.

\subsection{Motivation}

Even if a user does not have access to WLAN service, one of its neighbors might have. Multi-hop networking is an approach that allows a source node to use other nodes for relaying data to the destination node in multiple hops. Since multi-hop networking involves several other relaying nodes besides the source and destination nodes, many issues such as route selection and maintenance, QoS provisioning, and cooperative forwarding need to be addressed. Related work has investigated different network architectures and protocols for supporting VoIP service through multi-hop networking [7-9].

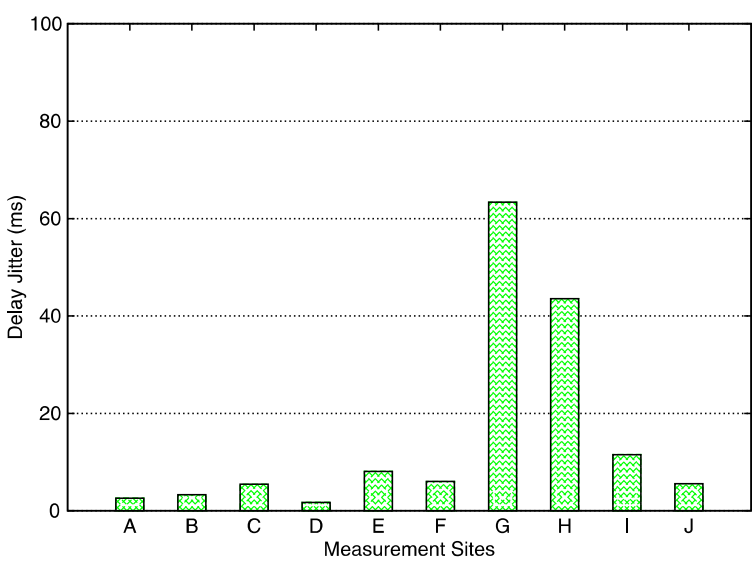

(b) Delay Jitter

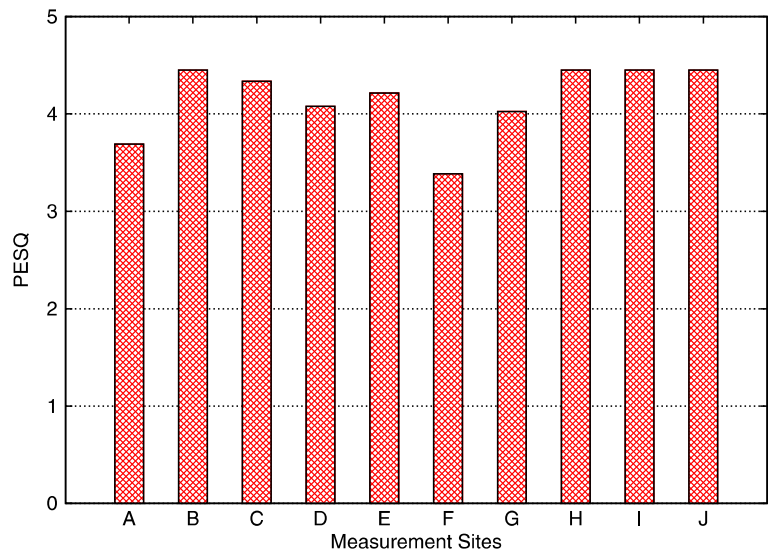

(d) Speech Quality (PESQ)

Fig. 3. Baseline VoIP performance. 

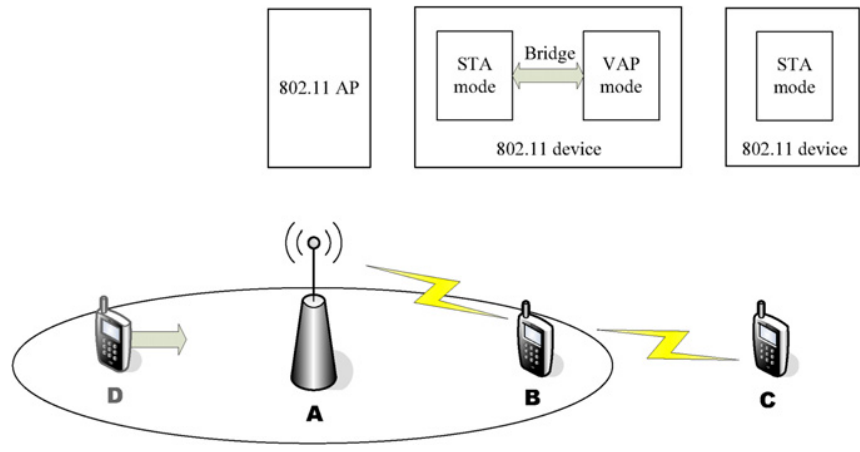

Fig. 4. One-hop extension.

In this paper we leverage the concept of multi-hop relay to address the problem of insufficient WLAN coverage. While any existing multi-hop routing protocol can be used in this context $[17,18]$, in this paper we adopt a simple approach for one-hop extension of the campus WLAN. The reasons for considering only one-hop extension in the target environment include:

1. While the WLAN considered does not have full coverage of the entire campus, it in fact has a relatively high deployment density (i.e. $80 \%$ in the NTU WLAN). Since a majority of the campus area is under WLAN service, it is expected that the number of hops required to connect a "coverage hole" to nearby access points is not large.

2. The WLAN considered is on a university campus, and the SIP phones need proper authentication and authorization to have access to the campus WLAN service. In one-hop extension, since the only relay node is still within the coverage of WLAN access points, it is easier to audit the behavior (and misbehavior) of the relay node for sake of security concern if necessary.

Limiting packet relay to one-hop extension of WLAN access points thus allows a minimum number of relay nodes to be involved and simplifies the protocol design. It has the additional benefits of avoiding performance instability and degradation due to data traversing a large number of hops as shown in related work $[2,19]$.

\subsection{Approach}

As mentioned in Section 2, it is possible that SIP phones are pre-configured with the necessary functionality for communication on campus. We discuss in the following the proposed approach to be implemented on SIP phones for one-hop extension of the campus WLAN.

In an 802.11 WLAN, a station needs to authenticate and associate with a chosen access point before it can use the WLAN service (e.g. for data transmission to or from the infrastructure). Any communication with the infrastructure or with any other 802.11 stations in the network has to go through the access point. Now consider the case with partial WLAN coverage as shown in Fig. 4. In the figure, station $C$ is outside the coverage of access point $A$, and hence it cannot get associated for WLAN service. If, on the other hand, station B can play the role of an access point, station $C$ can operate as usual for association with station $B$, and request for WLAN service from station $B$. Essentially, station B plays the role of a station (STA mode) and a virtual access point (VAP mode). Any MAC frame received through the VAP mode on station B is bridged to its STA mode for delivery to access point $A$. In this way, station $C$ can have access to the WLAN infrastructure as any other stations do, and the WLAN service provided by access point $A$ is virtually extended to station $C$. Note that to support both the STA mode and the VAP mode does not necessarily require station $B$ to be equipped with two 802.11 radios. Existing 802.11 device drivers allow one 802.11 radio to be "virtualized" into multiple modes of operation (through, say, time sharing of the radio resource). ${ }^{2}$ Therefore, it is possible to support the concept of the virtual access point without requiring any additional hardware at the relay node.

Note that creating the VAP mode instead of the AD-HOC mode (where station $B$ acts as a relaying peer) on station $B$ simplifies the operation of one-hop extension. Consider a scenario where station D (in the normal STA mode) is initially within the coverage of access point A as shown in Fig. 4. During the course of mobility, it moves toward the location of station $C$ and finally goes beyond the coverage of the WLAN. It then needs to rely on station B for packet relay similar to the case of station $C$ mentioned earlier. If station B operates in the VAP mode, then as far as station $\mathrm{D}$ is concerned, switching from one-hop communication (direct access) to two-hop communication (through station B) with access point A involves only a handoff between two access points since station B behaves like an access point. If station $\mathrm{B}$ operates in the AD-HOC mode, on the other hand, station $\mathrm{D}$ will need to change from the STA mode to the AD-HOC mode for communication with station $B$, thus incurring more overheads. The advantage of using the VAP mode can also been observed in a similar scenario where station $B$ moves when serving as the relay between station $D$ and access point $A$. In this case, station $\mathrm{D}$ can detect the failing link through the decreasing signal strength of station $B$ that falls below a predefined

\footnotetext{
2 The open-source MadWiFi driver [20], for example, allows concurrent running in both AP and STA modes through creation of multiple virtual WLAN interfaces atop one 802.11 device.
} 
threshold. Station D can then search in its neighborhood for any access point (be it "real" or "virtual") to perform handoff. Existing algorithms for handoff among WLAN access points thus can be used by station $\mathrm{D}$, and the overheads with multi-hop routing protocols including route discovery and maintenance can be avoided in this context.

An important issue, however, that needs to be considered for the relay node (station $B$ ) is the problem of resource (e.g. channel bandwidth and battery power) sharing between the STA and VAP modes. To prevent degradation of its performance as a station, the relay node can control, based on the channel bandwidth and battery power available, the number of peer stations (e.g. station C) that it can act as the relay node through the association process. If the relay node determines that its relaying capacity has been reached, it can reject association requests from peer stations. It is also possible for the relay node to stop operating in the VAP mode (after proper disassociation process) if it suffers from significant resource shortage. As mentioned before, in one-hop extension of the campus WLAN, the relay node (station $B$ ) is still within the coverage of the WLAN access point (access point $A$ ). Hence the WLAN access point can be made aware of the stations that act as virtual access points. The list of available virtual access points can then be announced to all stations. Any station that moves outside the WLAN coverage can use such information to search for and associate with the candidate relay node. All information required for association with the relay nodes can also be pre-configured in each SIP phone.

We summarize the operations of the proposed one-hop extension from the following three aspects:

1. Access point selection: For any SIP phone that needs to search for a new access point (e.g. during initialization or handoff), it first scans for a campus WLAN access point (e.g. one with the campus network SSID). If any such access point is detected, it proceeds with the association procedure for WLAN service. Otherwise, it searches for a virtual access point with the pre-configured network SSID. The station proceeds with association with the virtual access point using pre-configured information (e.g. authentication key) if such a virtual access point exists. Otherwise, the one-hop extension approach fails to extend the campus WLAN to the target SIP phone. (The dual-mode communication approach proposed in Section 4 can then be used if so desired.)

2. Access point handoff: A SIP phone periodically measures the signal quality such as RSSI (Received Signal Strength Indication) of the associated access point and nearby access points. If the SIP phone is currently associated with a virtual access point, and a campus WLAN access point with better signal quality is found, it proceeds with the handoff process to the campus WLAN access point. On the other hand, if the signal quality of the currently associated access point drops below a threshold (or it fails the test of other sophisticated algorithms for handoff decision considering, say, quality of service), the SIP phone proceeds with the Access point selection operation for choosing the target access point. To avoid the occurrence of the ping-pong effect, the signal qual- ity used for handoff decision is based on the average RSSI measured for a preset duration of time. Other sophisticated algorithms considering the use of adaptive threshold and/or hysteresis level and access point preference for handoff decision can also be adopted $[21,22]$.

3. Relay node capability: For any SIP phone that is within the coverage of the campus WLAN, it decides based on its call quality and battery power whether it can act as a relay node. If so, it creates a VAP mode and behaves as a virtual access point using pre-configured parameters; otherwise, it maintains at behaving as a pure 802.11 station (STA mode only). For a relay node, whenever an association request from another SIP phone is received, it checks if it has the capability to support the relay. If so, it proceeds with the association procedure with the requesting SIP phone; otherwise, it rejects the association. When acting as relay for other SIP phones, the relay node periodically monitors its call quality and/or battery power. If it decides to cut back the load of relaying, it can send disassociation requests to a selected number of peer stations. Finally, to limit the relay to one hop, if the relay node moves outside the coverage of the campus WLAN, it sends out disassociation requests to all SIP phones that are associated with it.

\subsection{Results and observations}

We implement the proposed approach for one-hop extension of campus WLAN using off-the-shelf hardware and open-source software. The MadWiFi WLAN driver [20] is used to support concurrent operations of VAP and STA modes on one WLAN device when needed. For sake of performance comparison, the SIP user agent used will automatically play a voice stream of spontaneous speech when a VoIP call is established. The peer SIP UA then records the incoming voice for objective evaluation of the speech quality (using PESQ). Round-trip time and delay jitter are also measured when the VoWLAN call is in progress.

To keep the scope of this paper focused, we consider a VoWLAN call between one wired SIP phone and one wireless SIP phone (say station C) through one-hop or two-hop communication with a campus WLAN access point. In onehop communication, the wireless SIP phone is within the campus WLAN coverage, and hence it communicates directly with the campus WLAN access point. In two-hop communication, the wireless SIP phone is outside the campus WLAN coverage, but it can rely on another wireless SIP phone (say station B) for relay. We compare the delay, jitter, and speech quality at the wireless SIP phone (station C) for one-hop and two-hop scenarios. We also investigate the impact of relay on the quality of call at the relay node (station B). For lack of space, we only present results measured at one campus location (location D in Fig. 2).

Fig. 5a thus shows the delay (round-trip time) distribution of the VoWLAN call under one-hop communication, while Fig. 5b shows the distribution under two-hop communication. It can be observed that the two-hop scenario suffers from a larger delay and jitter due to the need for one more hop of wireless communication involving 


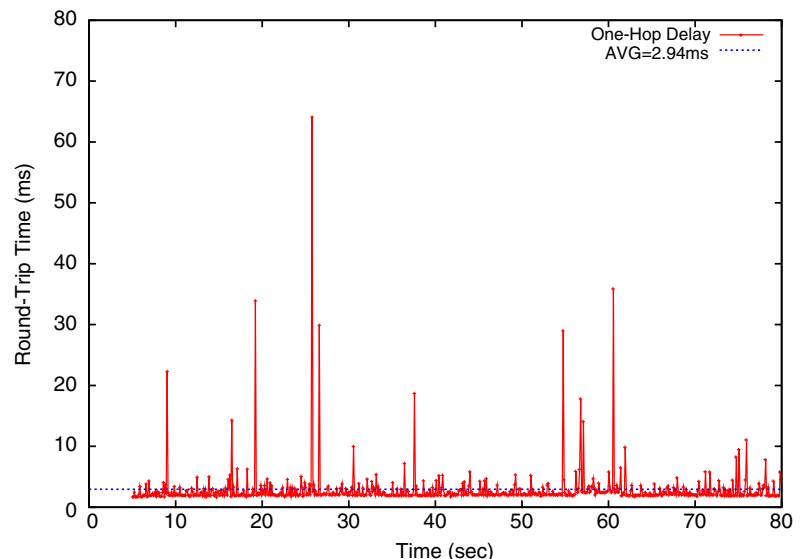

(a) One-Hop Delay

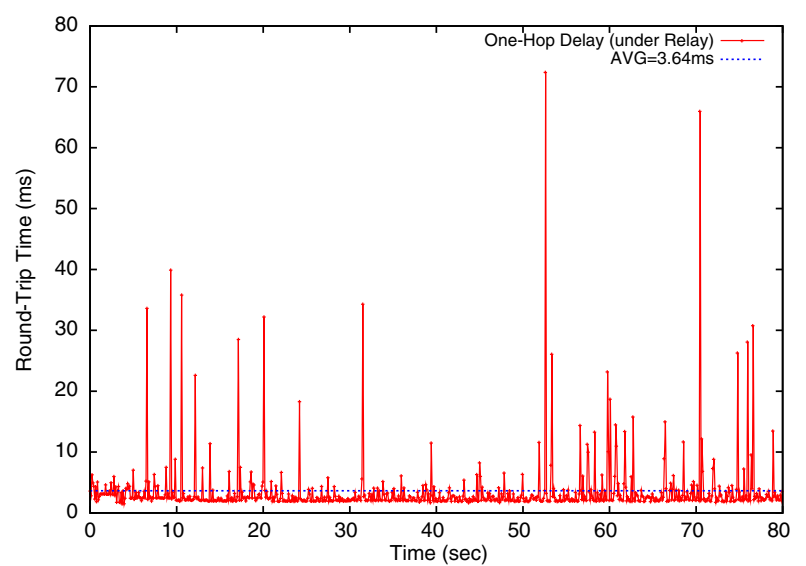

(c) One-Hop Delay (under Relay)

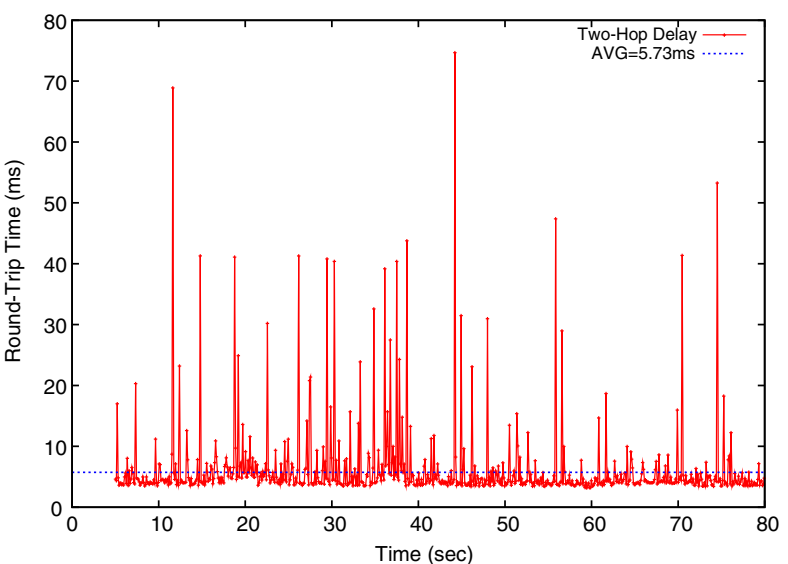

(b) Two-Hop Delay

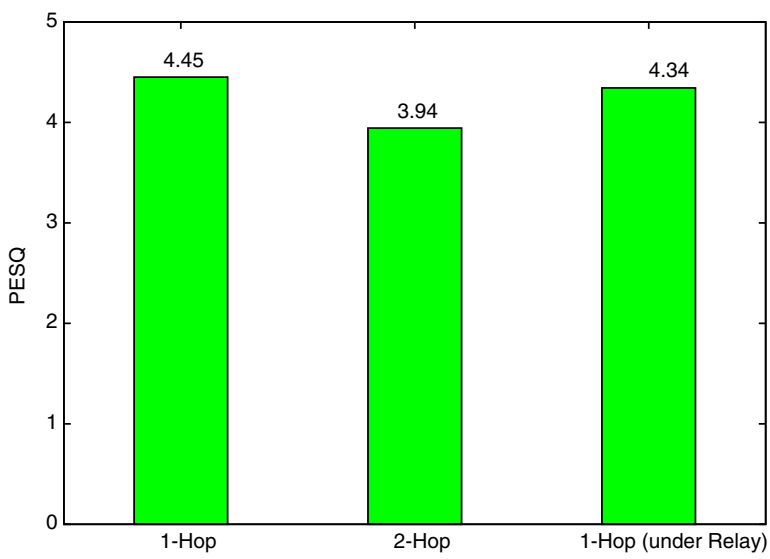

(d) Speech Quality

Fig. 5. Performance of one-hop extension.

random channel access. The increased delay and jitter introduce impairments to the voice, and hence the quality of speech. As shown in Fig. 5d, the objective speech quality (PESQ) drops from 4.45 for one-hop scenario to 3.94 for two-hop scenario.

While the proposed approach can provide acceptable speech quality for voice call placed outside the direct coverage of the campus WLAN, one issue that needs to be considered is the impact on the relay node. If the relay node is also in a VoWLAN call with a remote SIP phone, then the quality of the voice call might be affected due to the increased load of acting in the VAP mode. In Fig. 5c we show the observed delay and jitter at the relay node when it is relaying traffic for another SIP phone. Compared to Fig. 5a, it can be observed that the delay and jitter on average do increase at the relay node. The speech quality of the call at the relay node does decrease, but still falls in an acceptable range as observed in Fig. 5d.

To observe more closely the effect of relaying, we conduct another experiment with the two-hop scenario. In the experiment, the relay node (station B) initially acts as a pure wireless SIP phone without any responsibility of traffic relay for other SIP phones. During the progress of its voice call, a request for association and hence relay is received from another SIP phone (station $\mathrm{C}$ ). The relay node (station $\mathrm{B}$ ) honors the request and proceeds with the relay task for station C. Fig. 6 shows the delay measured at the relay node during the process. It can be ob-

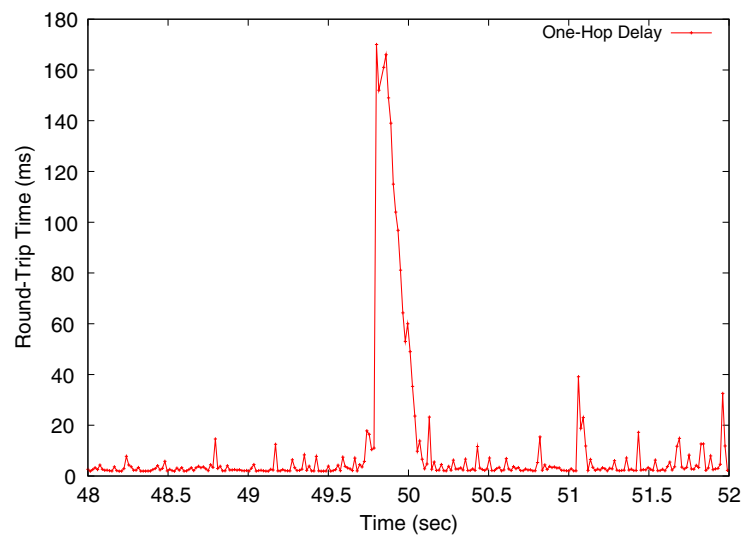

Fig. 6. Delay surge due to relay. 
served that after the request is received and station B starts to accept packets for traffic relay between the access point and station C (at about $49.5 \mathrm{~s}$ ), there is a transient surge in delay (the delay rises to $170 \mathrm{~ms}$ at $49.8 \mathrm{~s}$ ). The reason is due to the sudden change in channel condition for relaying a train of SIP packets for handshakes during the call setup process (recall that the voice call at station $C$ is about to be established then) [23]. While the delay surge does not last for long (for a duration of about $240 \mathrm{~ms}$ ), its magnitude is rather high that is unlikely to be absorbed by the typical de-jitter buffer. Therefore, RTP packets therein are discarded due to late arrivals. The effect is that an audio gap is incurred at station $B$ right after station $C$ connects to station $B$ for relay. The temporal discontinuity nonetheless introduces certain disruption to the voice call at station $B$ and should ideally be avoided. We discuss how this problem can be addressed in Section 5 .

\section{Dual-mode communication}

We have presented in Section 3 an approach that allows one-hop extension of the campus WLAN service area. While such an approach can effectively provide service to SIP phones not far away from the boundary of the service area, in some scenarios a relay for more than one hop may still be necessary. One approach, as we mentioned before, is to employ conventional multi-hop routing protocols and allow for relaying in more than one hop. In this section, however, we present a different approach that does not require multi-hop relay for serving users in WLAN dead spots. We first motivate the solution based on dualmode communication, and then present the proposed approach. Finally, we present evaluation results and some discussions.

\subsection{Motivation}

Existing mobile cellular telephony systems allow users to place or receive voice calls with practically "anywhere, anytime" coverage. IP telephony based on VoWLAN also aims at enabling such untethered communication while incurring significantly lower overheads and costs. The two telephony systems, while seemingly competitive against each other, can coexist and complement each other for providing better service to users. For example, whenever and wherever the VoWLAN service is available, users can use it for sake of reducing communication costs; at locations where VoWLAN cannot provide the desired service, users can switch to mobile cellular telephony (e.g. GSM) service for sake of maintaining the call reliability.

As simple as the concept is, however, the most challenging issue in using both GSM and VoWLAN is the need for seamless handoff of an active call between the two telephony systems when needed. Consider a voice call established by a mobile user through the VoWLAN service. During the course of mobility, the user moves out of the coverage of the WLAN service area (maybe even outside the one-hop extension region of the WLAN coverage using the approach in Section 3) and needs to switch to the GSM service for ensuring continuous voice communication. A handoff between the two telephony systems thus needs to be performed. If and only if the handoff between VoWLAN and GSM can take place automatically without disrupting the call in progress, the two telephony systems can synergistically provide the best service to the user.

To take advantage of the two telephony systems opportunistically without the need to bring two handsets, the concept of dual-mode handsets has emerged and come to the limelight. A key distinguishing feature of "dual-mode" handsets from conventional "multi-band" handsets is the ability to support voice communication through heterogeneous telephony technologies involving circuit-switched cellular telephony (such as GSM) and packet-switched Internet telephony (such as VoWLAN). Many GSM-WLAN, EDGE-WLAN, 3G-WLAN, and HSDPA-WLAN dual-mode handsets and smart phones have made their debut recently [24-26]. Such dual-mode handsets by design can be operated in any communication mode depending on service availability and user preference. Therefore, it is conceivable to have dual-mode handsets used as the wireless SIP phones on campus. In this case, it becomes an interesting issue to investigate the possibility of leveraging the service of existing mobile cellular telephony systems for improving the user experience when making VoWLAN calls. We discuss in the following how such dual-mode handsets can be used for enabling ubiquitous yet cost-effective voice communication in the campus WLAN.

\subsection{Approach}

A key challenge for dual-mode communication is seamless voice call continuity during handoff. As shown in Fig. 7, dual-mode handset $C$ is originally within the coverage of the WLAN access point, where the user places a VoWLAN call with the remote SIP phone. During the course of mobility, the user moves outside the WLAN coverage, and hence the call needs to be switched to the GSM system. While related work has proposed solutions that require the infrastructure support of the mobile cellular telephony system [10-13], in this paper we propose an end-to-end approach for vertical handoff on dual-mode handsets. The motivation is that, in the campus WLAN considered, it is easier to pre-configure SIP phones with the desired functionality than relying on infrastructure support from the telecom operator.

In the proposed approach, a Handoff Control software module is introduced atop the existing VoIP and GSM phone modules on the dual-mode handset as shown in Fig. 7. The VoIP UA and the GSM Phone modules are used by the dual-mode handset to make and receive calls through the WLAN and GSM modes respectively. The Handoff Control module is the core for handoff management. It controls when handoff should be triggered through feedback of the module that monitors the quality of the WLAN service (similar to that used in Section 3). The Handoff Control module also maintains states of the communications in progress including the identities (e.g. calling numbers) of the VoIP UA and GSM Phone modules. It interacts with the VoIP UA and GSM Phone modules through commands such as Call, Hangup, and Answer for transparent call migration between the two communication modes as we discuss in the following. 


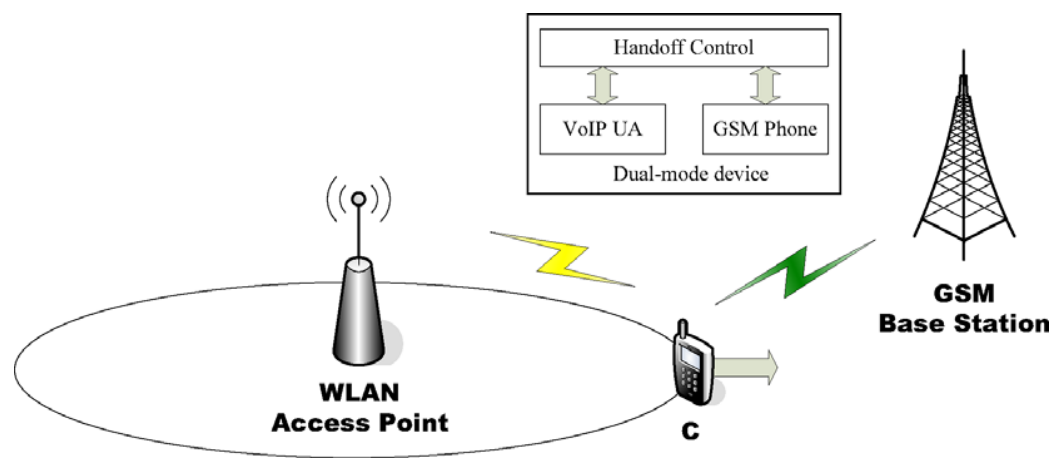

Fig. 7. Dual-mode communication.

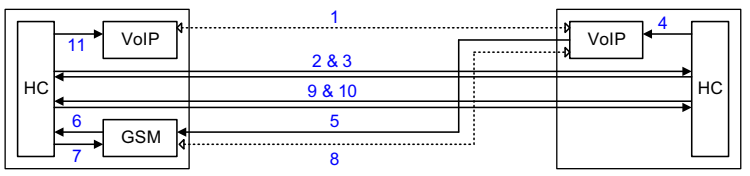

(a) WLAN to GSMH and off

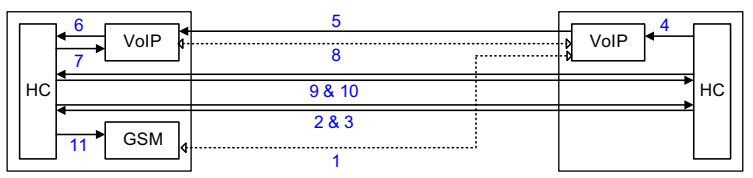

(b) GSM to WLAN Hand off

Fig. 8. Vertical handoff procedure on the dual-mode handset.

Consider first the case for WLAN to GSM handoff. The voice call between the dual-mode handset and the remote SIP phone is initially established through the VoWLAN service as indicated by "Step 1" in Fig. 8(a). Based on the feedback from the WLAN quality monitor module, the Handoff Control module decides to switch to the GSM service. It then sends a Handoff Trigger command to the remote peer to initiate handoff (as indicated by "Step 2"). The Handoff Trigger command includes in the message the state of the VoWLAN call (e.g. the call-ID in the SIP message header that uniquely identifies the call) and the GSM phone number so the remote peer can identify the dual-mode handset and match the command to the existing call session. Upon receiving the handoff trigger, the Handoff Control module on the remote peer responds with Handoff Trigger ACK ("Step 3"), and then directs its VoIP UA to make another call to the GSM phone number of the dual-mode handset ${ }^{3}$ (indicated by "Step 4" and "Step 5"). Note that the SIP-toPSTN (or SIP-to-GSM) call can be supported by the campus SIP infrastructure as shown in Fig. 1. Once the GSM Phone module on the dual-mode handset is alerted for the incoming call, the Caller ID is exposed to the Handoff Control module ("Step 6"). Based on its internal state, the Handoff Control

\footnotetext{
${ }^{3}$ Note that it is possible for the dual-mode handset to make the GSM call to the remote peer instead in consideration of some special GSM billing structure (where the caller pays for the entire communication cost). The decision as to whether the dual-mode handset or the remote peer makes the GSM call can be dynamically taken during the handshake process between the two Handoff Control modules (i.e. "Step 2" and "Step 3").
}

module matches the caller to the remote peer that the WLAN mode is currently in communication with, and then directs the GSM Phone module to answer the call using the Answer command ("Step 7"). After the call between the remote peer and the GSM mode is established and the voice stream is flowing between two ends ("Step 8"), the remote peer can then send a Handoff Complete command back to the dualmode handset ("Step 9"). The Handoff Control module on the dual-mode handset then responds with Handoff Complete ACK ("Step 10") and directs the VoIP UA module to hang up the call ("Step 11"), thus completing the handoff process from WLAN to GSM modes on the dual-mode handset.

Similarly, the case for GSM to WLAN handoff starts with the dual-mode handset being in a call with a remote SIP phone as indicated by "Step 1" in Fig. 8b. Once the WLAN quality monitor module notifies availability of the WLAN service, and the Handoff Control module decides to initiate the handoff, the Handoff Trigger command and acknowledgement are exchanged between the two ends through WLAN service ("Step 2" and "Step 3"). The Handoff Control module on the dual-mode handset conveys the identity of the VoIP UA (the "number" that the GSM Phone module dials) on the remote peer so the Handoff Control module on the remote peer can associate the incoming message with the existing call. The Handoff Control module on the remote peer directs the VoIP UA to make a call to the VoIP $U A$ on the dual-mode handset as shown in "Step 4" and "Step 5" of Fig. 8b. The required information for dialing (e.g. SIP URI of the dual-mode handset) is conveyed through the Handoff Trigger command sent earlier. The dual-mode handset can then match the Caller ID, and answers the call ("Step 6" and "Step 7"). After the new call (between two VoIP user agents) is established and the voice stream is flowing ("Step 8"), the old GSM call may be released through the Handoff Complete handshake as indicated in "Step 9," "Step 10," and "Step 11" in Fig. 8b.

It is clear that the proposed vertical handoff procedure achieves "make-before-break" soft handoff where the old and new voice streams overlap in time at the receiving end. Switching from the old call to the new call is performed after proper time alignment (using, say, cross correlation) of the two voice streams to ensure continuity of the call. Therefore, through handshakes between the Handoff Control modules at the two ends, vertical handoff between VoWLAN and GSM can be performed automati- 
cally and transparently as needed. From users' perspectives, they can enjoy ubiquitous voice communication through campus WLAN service even though the WLAN coverage may be spotty.

\subsection{Results and observations}

We implement the proposed approach for vertical handoff on the dual-mode handset using off-the-shelf hardware and open-source software. The handset is a GSM-WLAN dual-mode PDA equipped with the Intel PXA272 416MHz CPU and 64MB RAM. The dual-mode PDA runs the Windows Mobile 5.0 operating system, and the Windows Telephony API (TAPI) is used to control the GSM phone module. We port the open-source sipXtapi library [27] and use it to develop the VoIP UA for dialing and receiving VoWLAN calls.

To evaluate the performance of the proposed approach, we first use the dual-mode PDA to make voice calls to the remote SIP phone, and measure the call setup time. The call setup time is measured at the dual-mode PDA from the time the call is placed to the time the voice from the remote SIP phone is heard at the PDA. It can represent the delay between the time the handoff is triggered until the time the voice stream of the new call arrives. Fig. 9 thus shows the call setup times of VoWLAN and GSM calls for 11 iterations of experiments each.
As shown in Fig. 9, the call setup time for VoWLAN calls ranges from $1.21 \mathrm{~s}$ to $1.88 \mathrm{~s}$, with an average of $1.42 \mathrm{~s}$ over 11 iterations. For GSM calls, the call setup time ranges from $9.35 \mathrm{~s}$ to $9.68 \mathrm{~s}$ with an average of $9.51 \mathrm{~s}$. Since the call setup time is measured at the dual-mode PDA, it may involve delays due to the software and hardware processing time on the device. However, the primary reason for the prolonged delay between the GSM mode and the remote SIP phone is due to sophisticated signaling process and traversals of multiple networks involving the GSM access and core networks, PSTN network, Internet, and the campus network. Fig. 9 thus motivates the need for "make-before-break" soft handoff in the proposed approach to ensure voice call continuity during vertical handoff between VoWLAN and GSM.

Even though the proposed approach can ensure "makebefore-break" soft handoff, we have found through testbed experiments that handoff from VoWLAN to GSM is not always seamless. Rather, a temporal discontinuity in speech is perceived during the handoff process. To detail, we plot the waveform received at the dual-mode PDA during handoff as shown in Fig. 10. It can be observed from the figure that there is an audio gap of about $350 \mathrm{~ms}$ between the VoWLAN and GSM voice streams. Such an audio gap is, however, not observed for handoff from GSM to VoWLAN.

To understand the cause of the audio gap between the two voice streams, we measure the end-to-end delay of

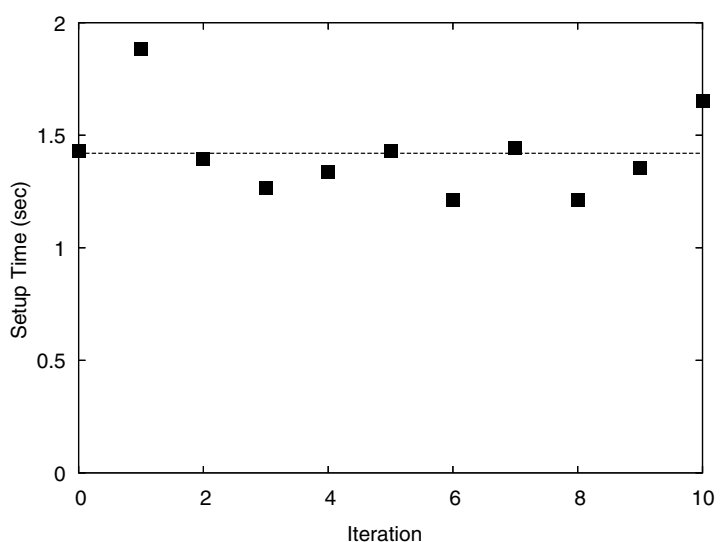

(a) VoWLAN Call to the SIP Phone

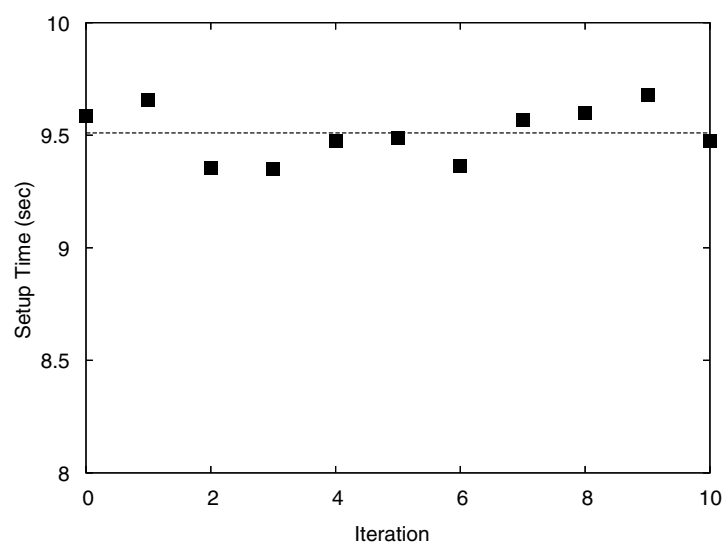

(b) GSM Call to the SIP Phone

Fig. 9. Call setup time.

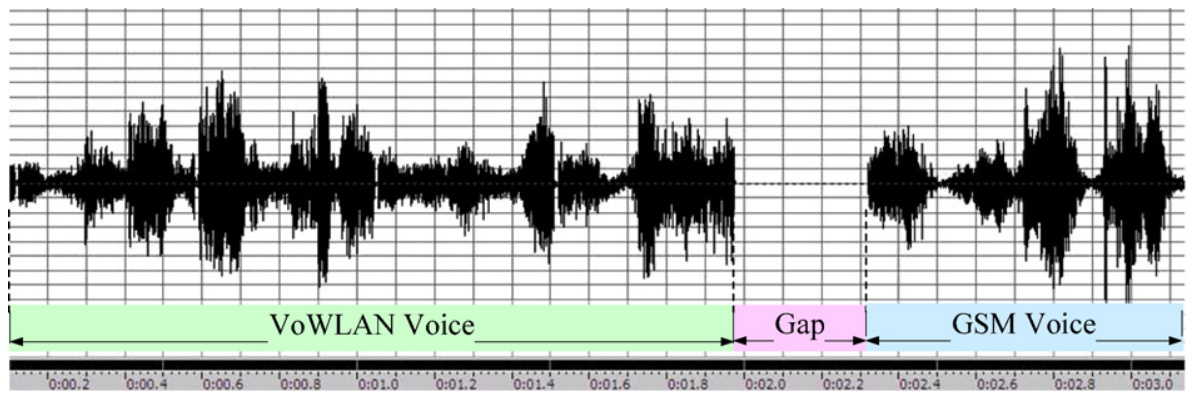

Fig. 10. Audio gap during VoWLAN-to-GSM handoff. 


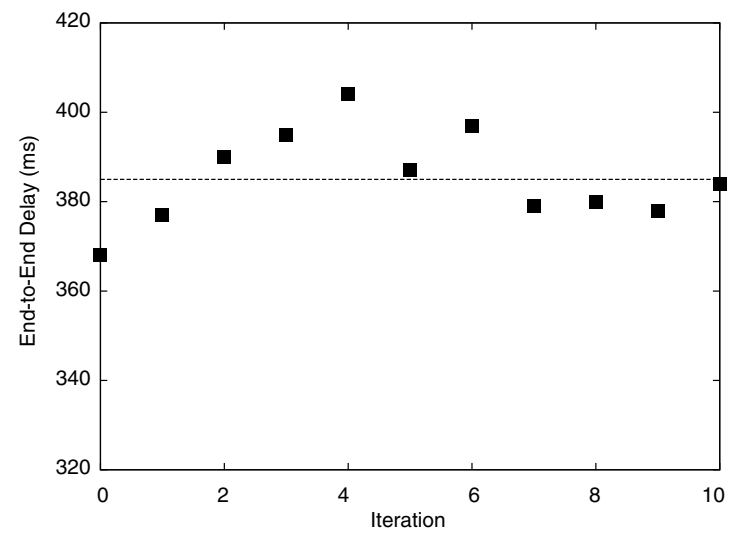

Fig. 11. End-to-end delay for GSM call.

the GSM call as shown in Fig. 11. The average delay as shown in the figure is $385 \mathrm{~ms}$ for GSM calls to the remote SIP phone. Compared to the case of the VoWLAN call shown in Fig. 3a, it can be observed that there is a significant delay mismatch between VoWLAN and GSM calls, with the latter experiencing longer delay than the former. As mentioned earlier, a time alignment process is performed to compensate for the delay mismatch of the new and old voice streams. In GSM-to-VoWLAN handoff, since the VoWLAN voice stream experiences a shorter delay, switching from GSM to VoWLAN can be done simply by delaying the VoWLAN voice stream in the buffer for an amount of time equal to the calculated time offset. In VoWLAN-to-GSM handoff, on the other hand, since the GSM voice stream experiences a longer delay, switching from VoWLAN to GSM introduces an artificial audio gap. In this way, a disruption due to temporal discontinuity is perceived in the voice call. We discuss in Section 5 how this problem can be addressed.

\section{Addressing temporal discontinuity}

As we have presented in Sections 3 and 4, one-hop extension and/or dual-mode communication can be employed in a campus WLAN environment to address the problem of spotty VoIP service due to partial WLAN coverage. As promising as the two proposed approaches are, however, they both suffer from the problem of temporal discontinuity in the voice call. In one-hop extension, the temporal discontinuity is introduced at the relay node due to the delay surge at the onset of relay. In dual-mode communication, on the other hand, the temporal discontinuity is introduced at the dual-mode handset for VoWLAN-toGSM handoff due to the delay mismatch between VoWLAN and GSM calls. It has been shown in [28] that temporal discontinuity impairments degrade the speech quality, and the larger the impairment magnitude (duration of the audio gap) is, the more severe the quality degradation is. Moreover, temporal discontinuity impairments introduce semantic and/or syntactic damages to speech, with the damages being more objectionable and annoying to native listeners than to non-native listeners (i.e. when the speech is understood by the listeners) [29]. As we have shown ear- lier, the duration of the temporal discontinuity is about $240 \mathrm{~ms}$ in one-hop extension and $350 \mathrm{~ms}$ in dual-mode communication. Therefore, temporal discontinuity should ideally be avoided to ensure seamless voice communication. In this section, we present a solution that can be used in tandem with one-hop extension and dual-mode communication to avoid the problem of temporal discontinuity.

\subsection{Time-scale modification of speech}

While temporal discontinuity is introduced in one-hop extension and dual-mode communication for different reasons in nature, one thing common is that the concerned speech segment is delayed instead of being lost completely. In one-hop extension, RTP packets are delayed due to the delay surge, while in dual-mode communication, the GSM voice stream is delayed due to the longer path. To address the problem of temporal discontinuity due to late arrivals, we adopt an approach based on time-scale modification of speech.

Time-scale modification is the process of changing the reproduction rate of a signal, including time-scale extension (slow down the playout speed) as well as time-scale compression (speed up the playout speed). Changing the playout speed exploits human's insensitivity to minor modulations in the speed of a speech signal [28]. Many algorithms have been proposed in the literature for performing efficient and high quality time-scale modification of speech [30-32]. In this paper, we choose the WSOLA (Waveform Similarity Overlap-Add) algorithm [32] for time-scale modification of speech since it preserves the pitch period and requires only time domain operations of the speech without any frequency domain transformation. More importantly, it can be applied to the speech synchronously on a frame-by-frame basis. In the following, we briefly discuss the operations of the WSOLA algorithm.

Let $x(n)$ be the original speech signal, and $y(n)$ be the time-scaled version of $x(n)$. Let $\Theta(n)$ be the linear time warping (scaling) function specifying that the sample occurring at $n$ in $x(n)$ should occur at $\Theta(n)$ in $y(n)$. For example, a linear time scaling operation uses $\Theta(n)=\beta n$ with $\beta<1$ for compression and $\beta>1$ for extension of the original signal. As shown in Fig. 12, the operation of the WSOLA algorithm is based on overlap-add-synthesis that consists of cutting out segments $k=0,1,2, \ldots$ of length $W$ using a window function $w(n)$ from the input signal $x(n)$ around analysis instants $\Theta^{-1}\left(S_{k}\right)$ and repositioning them at corresponding synthesis instants $S_{k}$ before adding them together to form the output signal $y(n)$. To maintain maximal local similarity to the original signal $x(n)$ across segment joins in the synthetic signal $y(n)$, WSOLA introduces the parameter $\delta_{k}$. The $k$ th synthesis segment, for example, is found by searching in $x(n)$ over a tolerance region $\left[-\delta_{\max }, \delta_{\max }\right]$ around $\Theta^{-1}\left(S_{k}\right)$ that has maximal similarity to the $(k-1)$ th synthesis segment right-shifted in time by $S_{k}-S_{k-1}$. In this way, it is ensured that the new synthesis segment will form a natural continuation of the previously chosen segment.

Typically, the synthesis instants are regularly spaced with $S_{k}=k S$, the window function is a Hann window with $50 \%$ overlap between successive segments $(W=2 S)$, and a 


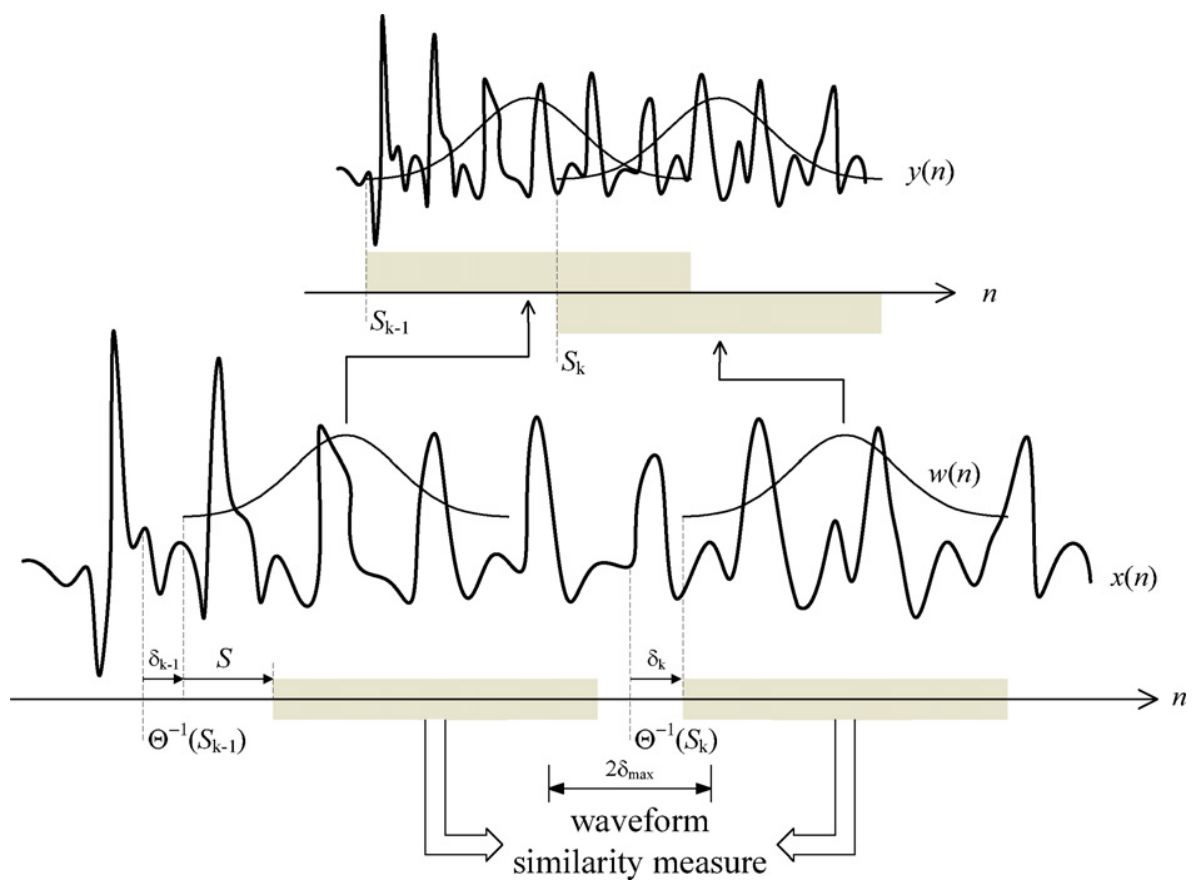

Fig. 12. Operation of WSOLA.

linear scaling function with $\Theta(n)=\beta n$ is used for the WSOLA algorithm. Then, $y(n)$ can be expressed as

$$
\begin{aligned}
& y(n)= w^{2}(n-(k-1) S) x\left(n+\frac{(k-1) S(1-\beta)}{\beta}+\delta_{k-1}\right) \\
&+w^{2}(n-k S) x\left(n+\frac{k S(1-\beta)}{\beta}+\delta_{k}\right) \\
& \text { for } \quad k S \leqslant n<(k+1) S .
\end{aligned}
$$

It is clear that the time-scaled signal $y(n)$ can be obtained from $x(n)$ synchronously in a left-to-right, frame-by-frame fashion.

For lack of space, we refer interested readers to [33] for detailed explanation and performance evaluation of the WSOLA algorithm.

To see how the WSOLA algorithm can be used in tandem with the proposed approaches, consider first the case of one-hop extension. Assume that a wireless SIP phone capable of acting as a virtual access point is in a voice call with the remote SIP phone. After it receives and decides to honor the association request from another SIP phone, it starts to monitors the buffering delay of its own playout buffer. If packets start to arrive late, the buffering delay of packets in the playout buffer will start to decrease. Whenever the buffering delay falls below a threshold (i.e. "safe margin"), the virtual access point starts to extends the playout speed of its audio frames to compensate for the reduction. The goal is to adjust the packet playout schedule such that the delay surge can be absorbed without packets being dropped due to late arrivals. It has to be noted, however, that since the magnitude of the delay surge may vary depending on the load of the relay node, the playout schedule needs to be dynamically adapted (i.e. the extension ratio may vary across audio frames). This adaptive playout scheduling mechanism is similar to re- lated work proposed for concealment of packet losses due to network congestion [34,35]. For the case of dualmode communication, assume that the new voice stream has been matched to the new voice stream and found to be lagging in time. The old voice stream is then extended in time to fill the delay mismatch (audio gap) between the two voice streams. The new voice stream is kept in the buffer until the playout schedule of the old voice stream matches that of the new voice stream, after which the voice call is switched to the new voice stream to ensure seamless transition of voice call.

\subsection{Performance evaluation}

We implement the proposed solution based on the open-source software for time-scale modification of speech [36]. We apply the time-scaling algorithm to reduce the audio gap in the voice call as shown in Fig. 13. A scaling factor of 1.25 is used to extend a speech segment of $1.2 \mathrm{~s}$ to mitigate an audio gap of $300 \mathrm{~ms}$. As shown in the figure, the time-scaling algorithm can effectively eliminate the impairment due to temporal discontinuity at the expense of introducing the impairment of time-scale modification to the original speech.

To investigate the performance of the time-scaling algorithm, and the tradeoff between the temporal discontinuity impairment and the time-scale modification impairment, we conduct an experiment surveying 30 listeners. Each listener is presented with two voice streams, one with an audio gap of about $300 \mathrm{~ms}$, and the other with the gap compensated by the time-scaling algorithm. The experiment is conducted as follows: first, the listener is presented with the processed voice stream and is given the hint that the voice may have time-scale modification. 


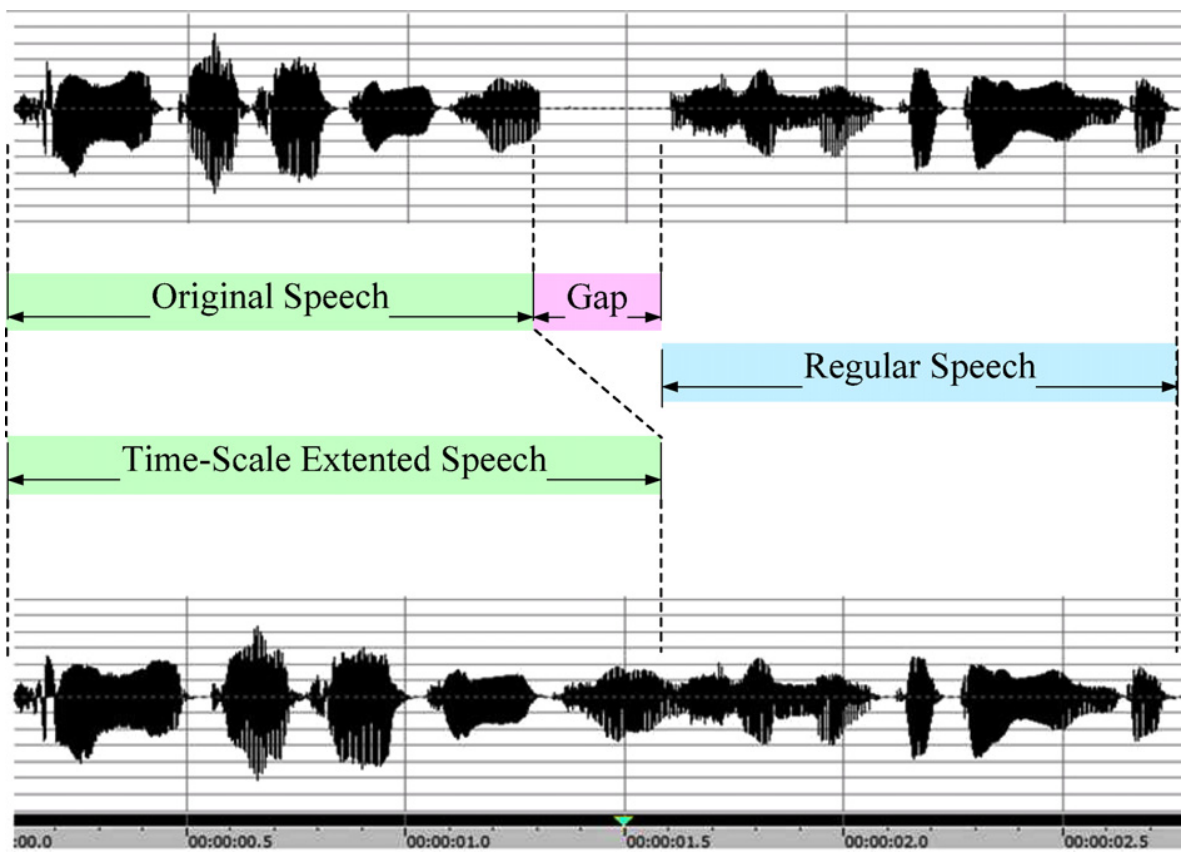

Fig. 13. Time-scale modification of speech.

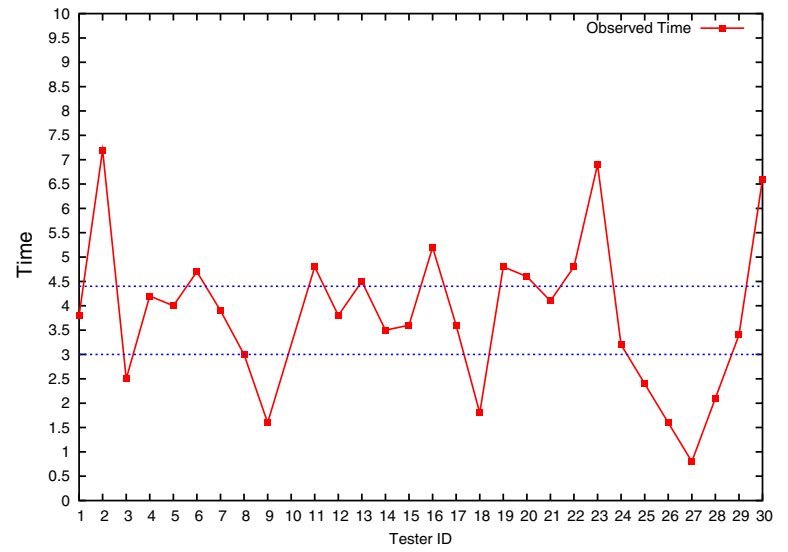

(a) Ability to Identify Impairment

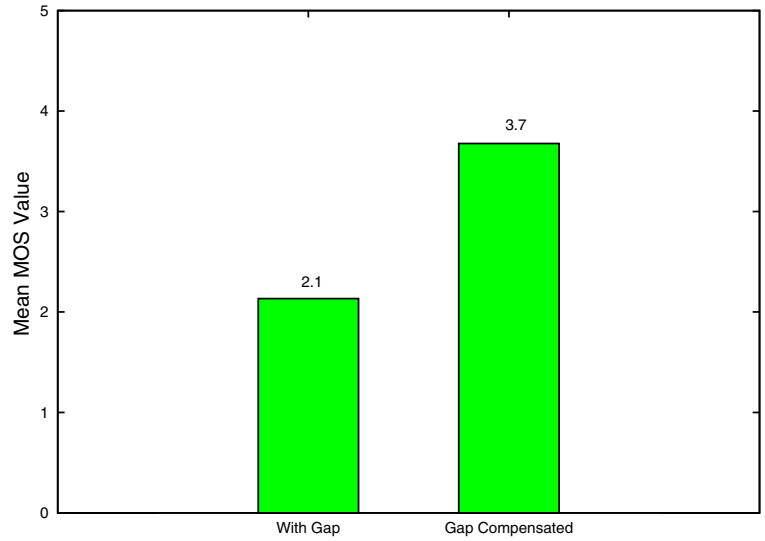

(b) Mean Opinion Score

Fig. 14. Subjective quality test.

The listener is then asked to identify the location of such temporal impairment within the voice stream. Finally, the listener is presented with the original voice stream (before transmission), and is asked to give a score from 1 to 5 for the two voice streams with artifacts (one with temporal discontinuity and one with time-scale modification). Fig. 14 thus shows the results of the tests. The time-scale modification is performed from $t=3 \mathrm{~s}$ to $t=4.5 \mathrm{~s}$. It can be observed from Fig. 14a that most listeners are not able to correctly identify the onset of the time-scale modification. In fact, many listeners mistakenly identify natural prosody of speech as artificial time-scale modification. The result that the voice stream with gap compensated gets a higher MOS value than the one with clear temporal gap as shown in Fig. 14b substantiates the argument that it is desirable to remove the temporal discontinuity for a more pleasing experience during the voice communication.

\section{Conclusion}

In this paper, we investigate the problem of supporting ubiquitous voice communication in a WLAN with spotty service area. We consider a university campus that has an established infrastructure for supporting SIP-based VoIP service, but the campus WLAN service does not have $100 \%$ full coverage. We propose two approaches called one-hop extension and dual-mode communication to enhance VoIP service in the target campus WLAN. The first approach uses the concept of virtual access points to extend the WLAN coverage, while the second approach leverages the avail- 
ability of dual-mode handsets for ubiquitous voice communication. We implement the two approaches, and evaluate their performance in the campus testbed environment. Evaluation results show that the proposed approaches can effectively enable voice communication in WLAN dead spots, but they have one common problem as the potential lack of support for voice call continuity. We then propose a cross-layer solution based on time-scale modification of speech to address the problem. Testbed evaluation shows promising results for using the proposed solutions to enable ubiquitous and seamless voice communication in a campus WLAN with partial coverage.

\section{Acknowledgements}

We would like to thank the Computer and Information Networking Center at National Taiwan University for information on the campus network infrastructure. We would also like to thank Yi-Yo Lin, Kuan-Lin Kuo, Chung-Wei Li, Tsung-Lin Tsai, Shuo-Wei Liao, and Yu-Wen Chen at the TONIC laboratory for their assistance and precursory research on this work. This work was supported in part by funds from the Taiwan Network Information Center (TWNIC) and the Excellent Research Projects of National Taiwan University under Grant 95R0062-AE00-04.

\section{References}

[1] S. Xu, T. Saadawi, Revealing the problems with 802.11 medium access control protocol in multi-hop wireless ad hoc networks, Elsevier Computer Networks Journal 38 (4) (2002) 531-548.

[2] P.-C. Ng, S.-C. Liew, Re-routing instability in IEEE 802.11 multi-hop ad-hoc networks, in: Proceedings of IEEE LCN, Tampa, FL, USA, November 2004.

[3] L. Huang, T.-H. Lai, On the scalability of IEEE 802.11 ad hoc networks, in: Proceedings of ACM MobiHoc, Lausanne, Switzerland, September 2002.

[4] A. Mishra, N. Petroni, W. Arbaugh, T. Fraser, Security issues in IEEE 802.11 wireless local area networks: a survey, Wireless Communications and Mobile Computing 4 (8) (2004) 821-833.

[5] Wireless Taipei - WIFLY [Online], Available at: <http://www.wifly. com.tw/>.

[6] Seattle Wireless [Online], Available at: <http://www.seattlewireless. net/>.

[7] S. Armenia, L. Galluccio, A. Leonardi, S. Palazzo, Transmission of VoIP traffic in multihop ad hoc IEEE 802.11b networks: experimental results, in: Proceedings of IEEE Wireless Internet (WICON), Budapest, Hungary, July 2005.

[8] H. Wu, X. Wang, Y. Liu, Q. Zhang, Z.-L. Zhang, SoftMAC: Layer 2.5 MAC for VoIP support in multi-hop wireless networks, in: Proceedings of IEEE SECON, Santa Clara, CA, USA, September 2005.

[9] R. Kortebi, D.-E. Meddour, Y. Gourhant, N. Agoulmine, SINR-based routing in multi-hop wireless networks to improve VoIP applications support, in: Proceedings of IEEE CCNC, Las Vegas, NV, USA, January 2007.

[10] M. Buddhikot, G. Chandranmenon, S. Han, Y.-W. Lee, S. Miller, L. Salgarelli, Design and implementation of a WLAN/CDMA2000 interworking architecture, IEEE Communications Magazine 41 (11) (2003) 90-100.

[11] H.-H. Choi, O. Song, D.-H. Cho, A seamless handoff scheme for UMTS-WLAN interworking, in: Proceedings of IEEE Globecom, Dallas, TX, USA, November 2004.

[12] Third Generation Partnership Project (3GPP), Unlicensed mobile access (UMA); architecture (stage 2), UMA Technical Specification R1.0.4, May 2005.

[13] Third Generation Partnership Project (3GPP), Generic access to the A/Gb interface; stage 2, 3GPP TS 43.318 V6.7.0, July 2006.

[14] SIPfoundry, sipXezPhone - a new sipXtapi based SIP user agent [Online], Available at: <http://www.sipfoundry.org/sipXezPhone/>.
[15] ITU-T, Perceptual evaluation of speech quality (PESQ): an objective method for end-to-end speech quality assessment of narrow-band telephone networks and speech codes, ITU Recommendation P.862, Feburary 2001.

[16] ITU-T, The E-model: a computational model for use in transmission planning, ITU Recommendation G.107, December 1998.

[17] C. Perkins, E. Royer, S. Das, Ad hoc on-demand distance vector (AODV) routing, IETF RFC 3561, July 2003

[18] T. Clausen, P. Jacquet, Optimized link state routing protocol (OLSR), IETF RFC 3626, October 2003.

[19] Y. Gourhant, F. Jan, T. Rasheed, R. Kortebi, R. Narayanan, Lessons gained from test beds of ad hoc networks and perspectives, in: Proceedings of ACM RealMAN, Florence, Italy, May 2006.

[20] MadWifi.org, Creating multiple interfaces [Online], Available at: <http://madwifi.org/wiki/UserDocs/MultipleInterfaces>.

[21] N. Zhang, J. Holtzman, Analysis of handoff algorithms using both absolute and relative measurements, IEEE Transactions on Vehicular Technology 45 (1) (1996) 174-179.

[22] W.-I. Kim, B.-J. Lee, J.-S. Song, Y.-S. Shin, Y.-J. Kim, Ping-pong avoidance algorithm for vertical handover in wireless overlay networks, in: Proceedings of IEEE VTC Fall, Baltimore, MD, USA, September 2007.

[23] J. Rosenberg, H. Schulzrinne, G. Camarillo, A. Johnston, J. Peterson, R. Sparks, M. Handley, E. Schooler, SIP: Session Initiation Protocol, IETF RFC 3261, June 2002

[24] Apple Inc., iPhone, 2007 [Online], Available at: <http:// www.apple.com/iphone/>.

[25] HTC Corporation, Dopod 818 Pro, 2006 [Online], Available at: <http://www.htc.com>.

[26] HTC Corporation, CHT9100, 2006 [Online], Available at: <http:// www.htc.com>

[27] SIPfoundry, sipXtapi - user agent framework SDK [Online], Available at: <http://www.sipfoundry.org/sipxtapi.html>.

[28] S. Voran, Perception of temporal discontinuity impairments in coded speech - a proposal for objective estimators and some subjective test results, in: Proceedings of International Conference on Measurement of Speech and Audio Quality in Networks (MESAQIN), Prague, Czech, May 2003.

[29] D.-S. Kim, ANIQUE: an auditory model for single-ended speech quality estimation, IEEE Transactions on Speech and Audio Processing 13 (5) (2005) 821-831.

[30] R. Roucos, A. Wilgus, High quality time scale modification for speech, in: Proceedings of IEEE ICASSP, Tampa, FL, USA, April 1985

[31] D. Bigorgne et al., Multilingual PSOLA text-to-speech system, in: Proceedings of IEEE ICASSP, Minneapolis, MN, USA, April 1993.

[32] W. Verhelst, M. Roelands, An overlap-add technique based on waveform similarity (WSOLA) for high quality time-scale modification of speech, in: Proceedings of IEEE ICASSP, Minneapolis, MN, USA, April 1993.

[33] W. Verhelst, Overlap-add methods for time-scaling of speech, Elsevier Speech Communication Journal 30 (4) (2000) 207-221.

[34] Y. Liang, N. Farber, B. Girod, Adaptive playout scheduling and loss concealment for voice communication over IP networks, IEEE Transactions on Multimedia 5 (4) (2003) 532-543.

[35] H. Ilk, S. Guler, Adaptive time scale modification of speech for graceful degrading voice quality in congested networks for VoIP applications, Elsevier Signal Processing Journal 86 (1) (2006) 127139.

[36] M. Flax, MFFM time scale modification for audio, 2001 [Online], Available at: <http://mffmtimescale.sourceforge.net/>.

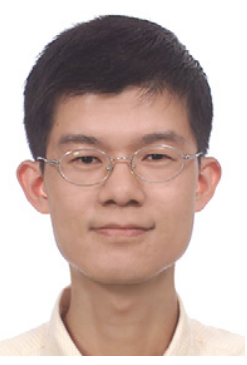

Hung-Yun Hsieh received the B.S. and M.S. degrees in electrical engineering from National Taiwan University, Taipei, Taiwan, ROC, and the Ph.D. degree in electrical and computer engineering from Georgia Institute of Technology, Atlanta, Georgia, USA. He joined the Department of Electrical Engineering and the Graduate Institute of Communication Engineering at National Taiwan University as an Assistant Professor in August 2004. His research interests include wireless communications and mobile computing. 


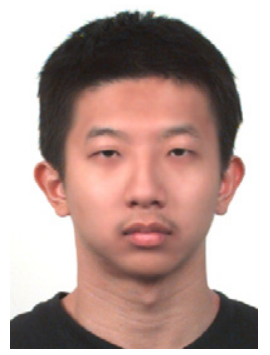

You-En Lin received the B.S. degree in electrical and control engineering from National Chiao Tung University, Hsinchu, Taiwan, ROC. He is currently pursuing the Ph.D. degree in the Graduate Institute of Communication Engineering at National Taiwan University, Taipei, Taiwan, ROC. His research interests are in areas of multi-hop wireless communication, vehicular ad hoc networks, and cognitive radio networks.

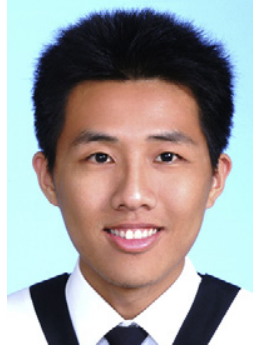

Hsiao-Pu Lin received the B.S. degree in electrical engineering from National Cheng Kung University, Tainan, Taiwan, ROC. He is currently pursuing the M.S. degree in the Graduate Institute of Communication Engineering at National Taiwan University, Taipei, Taiwan, ROC. His research is in the area of heterogeneous wireless networks, including vertical handoff and audio-video synchronization for dual-mode mobile devices. 